\title{
Nuclear reactions at intermediate energies
}

\author{
RADHEY SHYAM
}

Saha Institute of Nuclear Physics, 1/AF Bidhan Nagar, Kolkata 700064, India

\begin{abstract}
In the domain of Nuclear reactions at intermediate energies, the QCD coupling constant $\alpha_{s}$ is large enough $(\sim 0.3-0.5)$ to render the perturbative calculational techniques inapplicable. In this regime the quarks are confined into colorless hadrons and it is expected that effective field theories of hadron interactions via exchange of hadrons, provide useful tools to describe such reactions. In this contribution we discuss the application of one such theory, the effective Lagrangian model, in describing the hadronic reactions at intermediate energies whose measurements are the focus of a vast international experimental program.
\end{abstract}

In the quest for understanding the quantum chromodynamics (QCD), the hadron-hadron, hadron-photon and hadron-lepton reactions have played a major role. From these studies two quite distinct regimes of QCD have emerged. The first regime is of those processes that involve very high momentum transfers. Here the asymptotic freedom property of QCD has enabled the perturbative QCD (pQCD) methods to describe [1] a large set of high energy large momentum transfer reaction observables with great precision, providing the most precise test of QCD to date $[2,3]$.

The second regime that includes nuclear reactions at intermediate energies, is characterized by the confinement of quarks inside the hadrons. This remarkable feature, along with the running of the QCD coupling constant $\left(\alpha_{s}\right)$ towards larger values makes the pQCD methods inapplicable - one requires techniques that can solve the theory exactly. To date, the only fully predictive non-perturbative method for studying QCD at low energies is 
lattice QCD (LQCD). The progress in LQCD studies of the low-lying spectrum of QCD, hadronic structure and interactions has been significant in the recent years (see, eg., Ref. [4]).

Nevertheless, LQCD calculations are still far from being routinely amiable to solutions of intermediate energy nuclear reactions. It is therefore, necessary to use effective methods to describe the dynamics of such processes. The effective field theories where hadrons interact via exchange of hadrons, provide a viable alternative to LQCD for the description of the intermediate energy nuclear reactions (see, e.g., Refs. [5-11]). These theories implement the symmetries of the underlying theory, QCD, by using proper effective Lagrangians. However, many of these models invariably require $a$ priory knowledge of unknown parameters related to the coupling constants associated with the effective Lagrangians and the masses of the hadrons. Because the effective theories provide flexible theoretical approaches that enable a rapid understanding of the complexities involved in the scattering problems at intermediate energies and have the capability to guide to newer experimental studies, steady progress has been made towards determining the realistic input parameters involved in these theories.

In this presentation we discuss the application of effective-Lagrangian based theoretical models, to describe the meson production reactions (both non-strange and strange) in photon and meson induced reactions on nucleons at intermediate energies. We also present some results on the charmed baryon and meson production in the antiproton-proton annihilation process.

\section{Meson production in photon-nucleon reactions}

The photon induced reactions on nucleons where mesons and nucleons are measured as final decay products, have revealed a rich excitation spectrum of nucleons that reflects their complicated multi-quark inner dynamics. The determination of the properties of the nucleon excited states (or resonances) from the data of such experiments is a major challenge of hadron physics [12].

The effective methods that are used to describe the dynamics of meson production reactions, include explicitly the baryon resonance states whose properties are extracted by comparing the predictions of the theory with the experimental data [9-11,13-17]. However, for a reliable realization of this goal, a model is required that can analyze different reactions over the entire energy range using a single Lagrangian density, which generates all non-resonance contributions from Born, $u$ - and $t$-channel contributions without introducing new parameters. At the same time, the Lagrangian should 

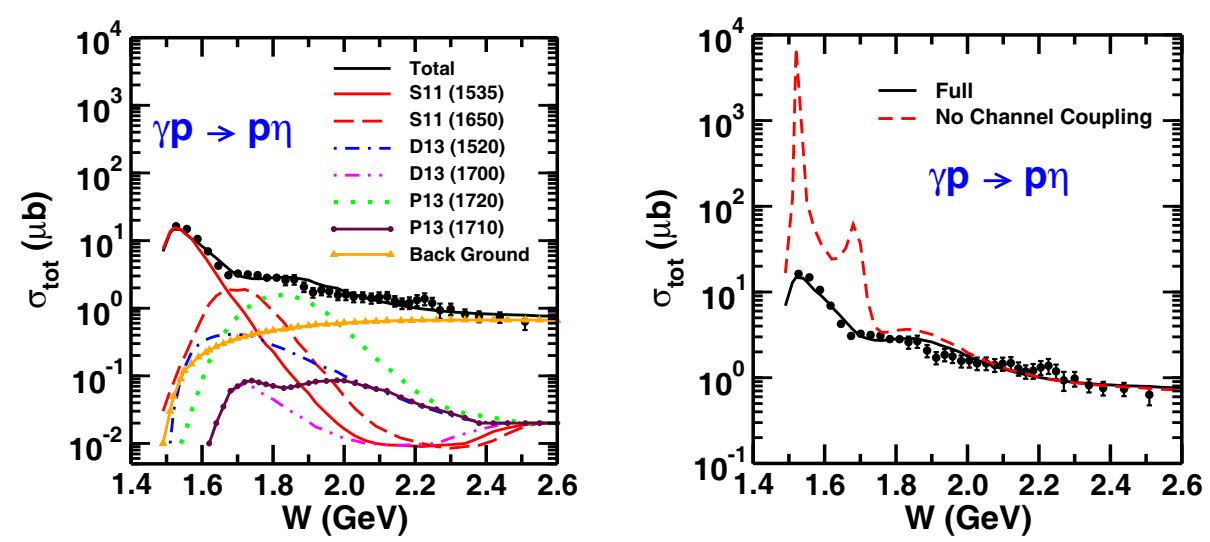

Figure 1: (Left panel) Total cross section for the $\gamma p \rightarrow \eta p$ reaction as a function of $\gamma p$ invariant mass $W$. Individual contributions of various resonances and of background terms are shown by different curves as indicated in the figure. (Right panel) Effect of channel coupling on the total cross section for the $\gamma p \rightarrow \eta p$ reaction as a function of $W$. The solid line represents the results of the full coupled-channel calculations while the dashed line shows the one where channel coupling is switched off.

also satisfy the symmetries of the fundamental theory (i.e. QCD) while retaining only mesons and baryons as effective degrees of freedom. The coupled-channels method within the $K$-matrix approximation is one model that respects these constraints while providing a way to analyze simultaneously all the reaction data for a multitude of observables in different reaction channels. It also leads to a convenient way of imposing the unitarity constraint because the $S$ matrix in this approach is unitary provided the $K$-matrix is taken to be real and Hermitian (see, e.g., Ref. $[11,16]$ ).

The experimental data on the $\eta$ meson and associated strangeness $\left(K^{+} \Lambda\right.$ and $K^{+} \Sigma^{0}$ ) production in photon-nucleon reactions are available for photon energies $\left(E_{\gamma}\right)$ in the range of threshold to $3 \mathrm{GeV}$. Thus they cover not only the entire resonance region but also the region where the background contributions ( $t$-channel amplitudes mainly) are expected to be dominant. The data for the $\eta$ channel are reviewed recently in Ref. [20]. The coupledchannels $K$-matrix method has been used extensively in Refs. [11,16] to describe the data of both these channels in the entire range of $E_{\gamma}$ with a single set of parameters. These reaction channels have also been studied within the Giessen coupled-channels $K$-matrix model in Refs. [18,19] for $E_{\gamma}$ between threshold to $2.0 \mathrm{GeV}$. 
As an example of the success of the coupled-channels $K$-matrix model of Refs. $[11,16]$ in describing the meson production in photon-nucleon reactions, we show in the left panel of Fig. 1 the contribution of various resonances to the total cross section $\left(\sigma_{t o t}\right)$ of the $\gamma p \rightarrow \eta p$ reaction. The experimental data are taken from Ref. [21]. We see that the general features of the data are described reasonably well by the calculations. While contributions of the $S_{11}(1535)$ resonance dominate the $\sigma_{\text {tot }}$ from near threshold to invariant mass $(W)$ values of $1.7 \mathrm{GeV}$ (corresponding to $\mathrm{E}_{\gamma} \sim 1.1 \mathrm{GeV}$ ), those of the $S_{11}(1650)$ and $P_{13}(1720)$ resonance are important for $\mathrm{E}_{\gamma}$ between $0.950 \mathrm{GeV}$ to $1.28 \mathrm{GeV}$ and $1.1 \mathrm{GeV}$ to $2.2 \mathrm{GeV}$, respectively. The contributions of other resonances are quite weak in the entire range of $E_{\gamma}$. The cross sections beyond $2 \mathrm{GeV}$ are mostly governed by the contributions of the background terms.

The effect of channel coupling on the $\sigma_{t o t}$ is shown in the right panel of Fig. 1. In the no coupling case (NCC), the amplitudes of various processes are simply added together, ignoring the modifications to the widths of the resonances introduced by the channel couplings. We notice that for $W>$ $1.8 \mathrm{GeV}$ the differences between the full and the NCC results are very small because the contributions of the resonances are almost negligible in this region. However, at lower energies, the channel-coupling effects are large. In fact, at some energies, the resonance propagators can develop poles in the absence of channel couplings. Thus modifications introduced to the widths of the resonances due to channel couplings are indeed vital for reproducing the energy dependence of the experimental cross sections. Therefore, the use of a full coupled-channels approach is essential to describe the mesonproduction in photo-nucleon reactions.

\section{Double strangeness $(S=-2)$ production with $K^{-}$induced reactions}

The investigations of the cascade $(\Xi)$ hypernuclei (having strangeness $S=$ $-2)$ are of great importance. The binding energies and widths of the $\Xi$ hypernuclear states determine the strengths of the $\Xi N$ and $\Xi N \rightarrow \Lambda \Lambda$ interactions, which are difficult to explore directly in a laboratory. This input is vital for understanding the multi-strange hadronic and quark matter. Since strange quarks are negatively charged they are preferred in charge neutral dense matter. Thus these studies are capable of probing the role of strangeness in the equation of state at high density, eg. in the cores of neutron stars [22]. 


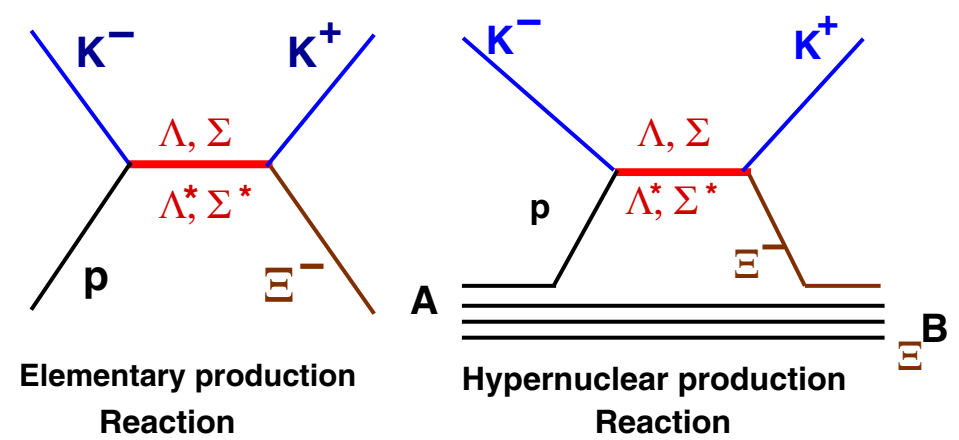

Figure 2: (Left panel) Graphical representation of the $s$-channel diagrams used to describe the $p\left(K^{-}, K^{+}\right) \Xi^{-}$reaction. The $u$-channel diagrams are not explicitly shown here but their contributions are included in the calculations. (Right panel) Diagram used to calculate the amplitudes of the $A\left(K^{-}, K^{+}\right) \Xi_{-} B$ reaction. $A$ represents the target nucleus and $\Xi_{-} B$ the final hypernucleus.

Because the $\left(K^{-}, K^{+}\right)$reaction leads to the transfer of two units of both charge and strangeness to the target nucleus, it provides one of the most promising ways of studying the $S=-2$ systems such as $\Xi$ hypernuclei and a dibaryonic resonance $(H)$, which is a near stable six-quark state with spin parity of $0^{+}$and isospin $0[23-25]$. The $\left(K^{-}, K^{+}\right)$reaction implants a $\Xi$ hyperon in the nucleus through the elementary $p\left(K^{-}, K^{+}\right) \Xi^{-}$process. The cross sections for this reaction were measured in the 1960s and early 1970s using hydrogen bubble chambers.

In Refs. [26,27], the $\left(K^{-}, K^{+}\right)$reaction on a proton target leading to the production of a free $\Xi^{-}$hyperon has been studies within a single-channel effective Lagrangian model (SCELM) (see, eg., Refs. [28, 29]). A coupledchannels study of this reaction is not feasible at this stage due to the scarcity of the data on the $K^{-}$interaction channels. In the SCELM the full field theoretic structure of the interaction vertices are retained and baryons are treated as Dirac particles. The initial state interaction of the incoming $K^{-}$ with a free or bound target proton leads to the excitation of intermediate $\Lambda$ and $\Sigma$ resonant states, which propagate and subsequently decay into $\Xi^{-}$ and $K^{+}$. In case of the reaction on nuclei, $\Xi^{-}$gets captured into one of the nuclear orbits, while the $K^{+}$meson goes out. We have considered as intermediate states the $\Lambda$ and $\Sigma$ hyperons and eight of their resonances with masses up to $2.0 \mathrm{GeV}$, which are represented by $\Lambda^{*}$ and $\Sigma^{*}$ in Figs. 2 . The effective Lagrangians and the coupling constants at various vertices are taken to be the same as those described in Ref. [26]. 

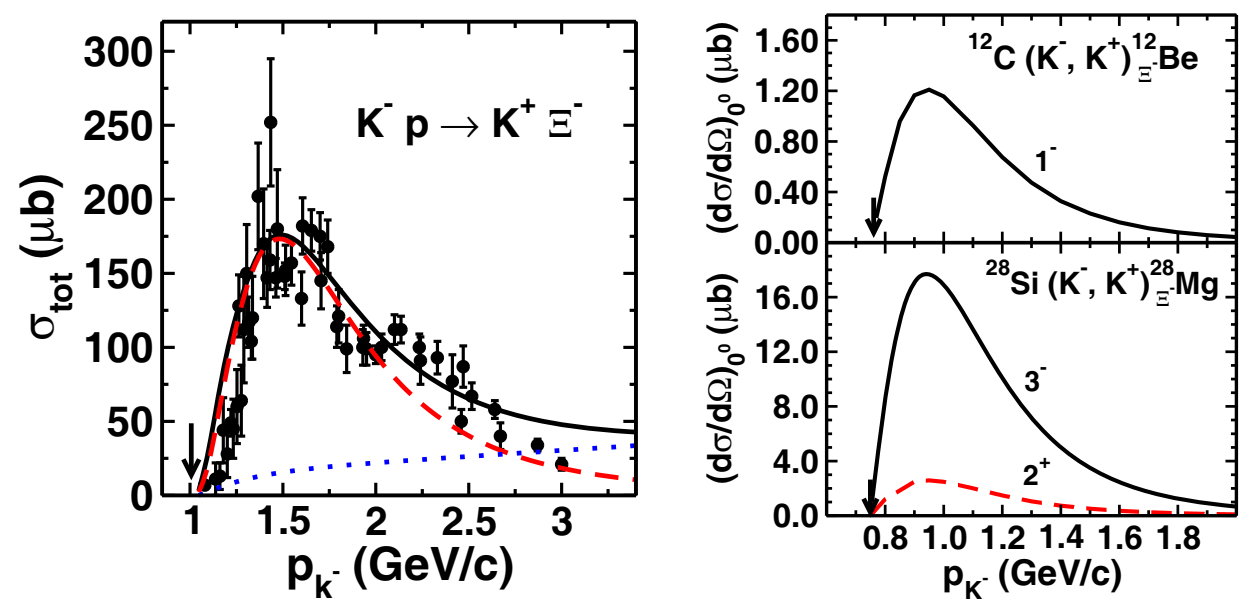

Figure 3: (Left panel) Total cross section for the $p\left(K^{-}, K^{+}\right) \Xi^{-}$reaction as a function of incident $K^{-}$momentum. Individual contributions of of $s$ - and $u$-channel diagrams to the total cross sections are also shown. (Right panel) $p_{K^{-}}$dependence of the zero degree differential cross section for the ${ }^{12} \mathrm{C}\left(K^{-}, K^{+}\right){ }^{12} \Xi-\mathrm{Be}$ and ${ }^{28} \mathrm{Si}\left(K^{-}, K^{+}\right)^{28} \Xi \mathrm{Mg}$ reactions. The spin-parity of the final hypernuclear states are indicated on each curve. Arrows show the thresholds for the corresponding reactions.

In the left panel of Fig. 3, we show comparisons of the SCELM calculations with the data for the $\sigma_{t o t}$ of the $p\left(K^{-}, K^{+}\right) \Xi^{-}$reaction as a function of $K^{-}$beam momenta $\left(p_{K^{-}}\right)$. It is clear that the data are reproduced reasonably well by the model within the statistical errors. The zero degree differential cross sections for the hypernuclear production reactions ${ }^{12} \mathrm{C}\left(K^{-}, K^{+}\right){ }^{12} \Xi^{-}$Be and ${ }^{28} \mathrm{Si}\left(K^{-}, K^{+}\right){ }^{28} \Xi_{-} \mathrm{Mg}$ calculated by using the same vertex parameters are shown in the right panel of Fig. 3. In these calculations we have have employed pure single-particle-single-hole $\left(p^{-1} \Xi\right)$ configurations to describe the nuclear structure part. The bound protonhole and $\Xi^{-}$-particle state spinors were generated in a phenomenological model, where they are obtained by solving the Dirac equation with scalar and vector fields having Woods-Saxon radial forms. With a set of radius and diffuseness parameters, the depths of these fields are searched to reproduce the binding energy (BE) of a given state. The BEs for the $\Xi^{-}$bound states were taken to be those predicted in the latest version of the quarkmeson coupling (QMC) model $[30,31]$ as their experimental values are still unknown.. In this figure, we have shown results for populating the hypernuclear state with maximum spin of natural parity for each configuration. We see that the zero degree differential cross sections for the $\Xi^{-}$hypernuclear 
production reactions peak around the beam momentum of $1.0 \mathrm{GeV} / \mathrm{c}$ with peak cross section of more than $1 \mu b$. They closely follow the trends of the elementary $\Xi^{-}$production cross sections. Our predictions will be useful for the future JPARC experiments.

\section{Charmed hadron production in $\bar{p} p$ annihilation}

The heavy flavored hadrons consisting of relatively heavier mass charm quarks provide an additional handle for the understanding of QCD. The large mass of the charm quark introduces a mass scale that is much larger than the confinement scale $\Lambda_{Q} \approx 300 \mathrm{MeV}$. In contrast, the energy scale of the lighter quarks is $\ll \Lambda_{Q}$. The presence of two scales in such systems naturally leads to the construction of an effective theory where one can actually calculate a big portion of the relevant physics using perturbation theory and renormalization-group techniques.

In this context, the investigations of the production of heavy flavor hadrons are of great interest. Since the discovery of $J / \psi$ in $1974[32,33]$, the production of charmonium $(c \bar{c})$ states has been extensively studied in electron-positron and proton-antiproton $(\bar{p} p)$ annihilation experiments. Yet a substantial part of the charmonium spectrum is still to be precisely measured. The first charmed baryon states were detected in 1975 in neutrino interactions [34]. Although many new excited charmed baryon states have been discovered since then, the studies of the production and spectroscopy of the charmed baryons have not been carried out in the same detail as the charmonium states.

In the near future, charmed hadron production will be studied in the $p \bar{p}$ annihilation in the $(\bar{P} A N D A)$ experiment at the FAIR in GSI, Darmstadt [35]. The advantage of using antiprotons in the study of the charmed baryon is that in $p \bar{p}$ collisions the production of no extra particle is needed for the charm conservation, which reduces the threshold energy. Moreover, all the the charmonium states can be reached directly in this method, which is in contrast to the electron-positron annihilation where direct formation of the only those final charmonium states is allowed that have the quantum numbers of the photon $\left(J^{P C}=1^{--}\right)$.

For the planning of these experiments at the $\bar{P} A N D A$ facility, reliable theoretical estimates of the cross section of $\bar{p} p \rightarrow \bar{\Lambda}_{c}^{-} \Lambda_{c}^{+}, \bar{p} p \rightarrow \bar{D}^{0} D^{0}$ and $\bar{p} p \rightarrow D^{-} D^{+}$reactions are of crucial importance [36,37]. In Ref. [38], the SCELM has been used to study the $\bar{p} p \rightarrow \bar{\Lambda}_{c}^{-} \Lambda_{c}^{+}$reaction as a sum of the $t$-channel $D^{0}$ and $D^{* 0}$ meson-exchange diagrams [Fig. 4(a)]. A similar model 


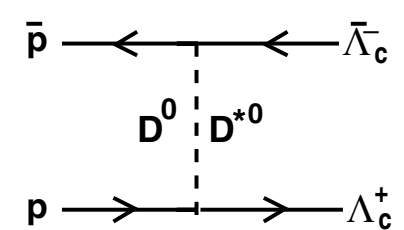

(a)

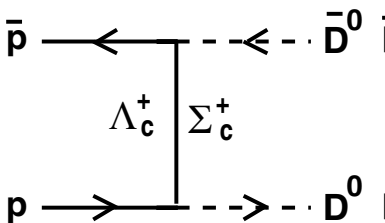

(b)

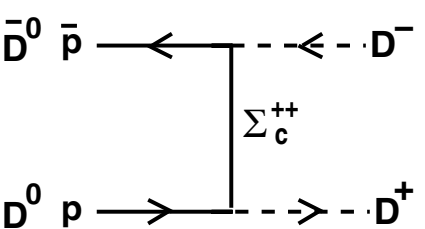

(c)

Figure 4: Graphical representations of the $t$-channel meson exchange diagrams used to describe the $\bar{p}+p \rightarrow \bar{\Lambda}_{c}^{-}+\Lambda_{c}^{+}$reaction (a), and the $t$-channel baryon exchange diagrams to describe the $\bar{p}+p \rightarrow \bar{D}^{0}+D^{0}$ and $\bar{p}+p \rightarrow D^{-}+D^{+}$reactions (b and c).

is employed in Ref. [39] to calculate the cross sections of $\bar{p} p \rightarrow \bar{D}^{0} D^{0}$, and $\bar{p} p \rightarrow D^{-} D^{+}$reactions as a sum of the $t$-channel $\Lambda_{c}^{+}$and $\Sigma_{c}^{+}$, and $\Sigma_{c}^{++}$ baryon-exchange diagrams, respectively [Figs. 4(b) and 4(c)]. Also included in these calculations are the $s$-channel excitation, propagation and decay of the $\Psi(3770)$ resonance into the $\bar{D}^{0} D^{0}$ and $D^{-} D^{+}$channels.

It is found that the $\sigma_{\text {tot }}$ of the $\bar{p} p \rightarrow \bar{\Lambda}_{c}^{-} \Lambda_{c}^{+}$and $\bar{p}+p \rightarrow \bar{D}^{0} D^{0}$ reactions at the $\bar{p}$ beam momentum of $15 \mathrm{GeV} / \mathrm{c}$ (which the regime of the $\bar{P} A N D A$ experiment) are around $25 \mu \mathrm{b}$ and $550 \mathrm{nb}$, respectively. These values are drastically larger than those predicted in previous calculations based on models that invoke the quark degrees of freedom. The future $\bar{P} A N D A$ experiment will clarify the adequacy of various models at these relatively lower beam momenta.

In summary, we can say that effective Lagrangian models are able to describe resonably well the intermediate energy nuclear reactions. The coupled-channels $K$-matrix method allows for the simultaneous calculation of the observables for a large multitude of reactions with considerably fewer parameters than would be necessary if each reaction channel were fitted separately. For the strangeness and charm production reactions, a singlechannel effective Lagrangian method with suitably chosen parameters is able to make important predictions that will be tested in the future experiments planned at upcoming facilities.

Acknowledgements: This work has been supported by the Helmholtz International Center (HIC) for FAIR and the Council of Scientific and Industrial Research (CSIR), India. The author acknowledges useful discussions with H. Lenske, O. Scholten, A.W. Thomas and K. Tsushima. 


\section{References}

[1] S. Bethke, Progr. Part. \& Nucl. Phys. 58 (2007) 351.

[2] John Collins, Foundations of Perturbative QCD, Cambridge, 2013.

[3] A.H. Müller, Phys. Rep. 73 (1981) 237.

[4] R.A. Briceno, Z. Davoudi, and Thomas C. Luu, arXiv:1406.5673 [heplat].

[5] M. Nielsen, F.S. Navarra and S.H. Lee, 2010 Phys. Rept. 497 (2010) 41.

[6] I.C. Cloet and C.D. Roberts 2014 Prog. Part. Nucl. Phys. 77 (2014) 1.

[7] J. de Vries, U.-G. Meissner, E. Epelbaum, N. Kaiser, Eur. Phys. J. A 49 (2013) 149.

[8] D. Siemens, V. Bernard, E. Epelbaum, H. Krebs, U.-G. Meissner, Phys.Rev. C 89 (2014) 6, 065211.

[9] T. Feuster and U. Mosel, Phys. Rev. C 58 (1998) 457; ibid. 59 (1999) 460.

[10] G. Penner and U. Mosel, Phys. Rev. C 66 (2002) 055211; ibid. 66 (2002) 055212 .

[11] R. Shyam and O. Scholten, Phys. Rev. C 78 (2008) 065201.

[12] S. Capstick and W. Roberts, Prog. Part. Nucl. Phys. 45 (2000) 241.

[13] B. Saghai and Z. Li, Eur. Phys. J. A 11 (2001) 217.

[14] B. Borasoy, E. Marco, and S. Wetzel, Phys. Rev. C 66 (2002) 055208.

[15] J. Durand, B. Julia-Diaz, T.-S.H. Lee, A. Matsuyama, T. Sato, Phys. Rev. C 78 (2008) 025204.

[16] R. Shyam, O. Scholten and H. Lenske, Phys. Rev. C 81 (2010) 015204.

[17] D. Rönchen, M. Döring, H. Haberzettl, J. Haidenbauer, U.-G. Meiner, K.Nakayama, Eur. Phys. J. A 51 (2015) 70.

[18] V. Shklyar, H. Lenske, and U. Mosel, Phys. Rev. C 87 (2013) 015201, and references of previous studies therein. 
[19] Xu Cao, V. Shklyar and H. Lenske, Phys. Rev. C 88 (2013) 055204.

[20] V.L. Kashevarov, arXiv:1506.02546 [nucl-ex].

[21] V. Crede et al., Phys. Rev. Lett. 94 (2005) 012004.

[22] J. Schaffner-Bielich, Nucl. Phys. A 804 (2008) 309; ibid. Nucl. Phys. A 835 (2010) 279c.

[23] R. L. Jaffe, Phys. Rev. Lett. 38 (1977) 195

[24] P. J. Mulders and A. W. Thomas, J. Phys. G 9 (1983) 1159.

[25] R. Shyam, O. Scholten and A.W. Thomas, Phys. Rev. C 88 (2013) 025209 .

[26] R. Shyam, O. Scholten and A. W. Thomas, Phys. Rev. C 84 (2011) 042201(R).

[27] B.C. Jackson, Y. Oh, H.Haberzettl, and K. Nakayama, Phys. Rev. C 91 (2015) 065208.

[28] R. Shyam, Phys. Rev. C 60 (1999) 055213.

[29] R. Shyam and U. Mosel, Phys. Rev. C 67 (2003) 065202.

[30] K. Tsushima, R. Shyam and A. W. Thomas, Few-Body Syst. 54 (2013) 1271.

[31] R. Shyam, Nucl. Phys. A 914 (2013) 79.

[32] J. Aubert et al., Phys. Rev. Lett. 33 (1974) 1404.

[33] J. Augustin et al., Phys. Rev. Lett. 33 (1974) 1406.

[34] E. Cazzoli, A. Cnops, P. Connolly, R. Louttit, M. Murtagh, R. Palmer, N. Samios, T. Tso, and H. Williams, Phys. Rev. Lett. 34 (1975) 1125.

[35] U. Wiedner, Prog. Part. Nucl. Phys. 66, 477 (2011).

[36] J. Haidenbauer, and G. Krein, Phys. Lett. B 678, 314 (2010).

[37] J. Haidenbauer, and G. Krein, Phys. Rev. D 89, 114003 (2014); ibid. 91, 114022 (2015).

[38] R. Shyam and H. Lenske, Phys. Rev. D 90 (2014) 014017.

[39] R. Shyam and H. Lenske, arXiv:1509.04786 [hep-ph]. 